\title{
Multiplicity results for biharmonic equations involving multiple Rellich-type potentials and critical exponents
}

\author{
Jinguo Zhang ${ }^{1}$ and Tsing-San Hsu ${ }^{2 *}$
}

\section{"Correspondence:}

tshsu@mail.cgu.edu.tw

${ }^{2}$ Department of Natural Science in the Center for General Education,

Chang Gung University, Taoyuan, Taiwan

Full list of author information is available at the end of the article

\begin{abstract}
In this paper, a biharmonic equation is investigated, which involves multiple Rellich-type potentials and a critical Sobolev exponent. By using variational methods and analytical techniques, the existence and multiplicity of nontrivial solutions to the equation are established.
\end{abstract}

MSC: 47G20; 35J50; 36B65

Keywords: Multiplicity; Biharmonic equations; Rellich-type potential; Critical Sobolev exponent

\section{Introduction}

In this paper, we study the following biharmonic equation:

$$
\begin{cases}\Delta^{2} u-\sum_{i=1}^{k} \frac{\mu_{i}}{\left|x-a_{i}\right|^{4}} u=|u|^{2^{*}-2} u+\lambda|u|^{q-2} u, & x \in \Omega, \\ u=\frac{\partial u}{\partial n}=0, & x \in \partial \Omega,\end{cases}
$$

where $\Omega \subset \mathbb{R}^{N}(N \geq 5)$ is a smooth bounded domain such that the different points $a_{i} \in \Omega$, $i=1,2, \ldots, k, k \geq 2, \frac{\partial}{\partial n}$ is the outward normal derivative, $0 \leq \mu_{i}<\bar{\mu}:=\left(\frac{N(N-4)}{4}\right)^{2}, \lambda>0$, $1 \leq q<2^{*}$, and $2^{*}:=\frac{2 N}{N-4}$ is the critical Sobolev exponent.

Equation $\left(E_{\lambda}\right)$ is related to the following Rellich inequality [22]:

$$
\int_{\Omega} \frac{u^{2}}{|x-a|^{4}} d x \leq \frac{1}{\bar{\mu}} \int_{\Omega}|\Delta u|^{2} d x, \quad \forall a \in \Omega, u \in H_{0}^{2}(\Omega),
$$

where $H_{0}^{2}(\Omega)$ is the completion of $C_{0}^{\infty}(\Omega)$ with respect to $\left(\int_{\Omega}|\Delta \cdot|^{2} d x\right)^{1 / 2}$. Then the following best constant is well defined:

$$
A_{\mu}(\Omega):=\inf _{H_{0}^{2}(\Omega) \backslash\{0\}} \frac{\int_{\Omega}\left(|\Delta u|^{2}-\mu \frac{u^{2}}{|x-a|^{4}}\right) d x}{\left(\int_{\Omega}|u|^{2} d x\right)^{\frac{2}{2^{*}}}}, \quad \forall a \in \Omega, \mu<\bar{\mu} .
$$

Note that it is well known that $A_{\mu}(\Omega)$ is independent of $\Omega$ and that $A_{\mu}(\Omega)$ is not obtained except in the case with $\Omega=\mathbb{R}^{N}$. Moreover, the minimizers of $A_{\mu}(\Omega)$ have been investi-

(c) The Author(s) 2019. This article is distributed under the terms of the Creative Commons Attribution 4.0 International License (http://creativecommons.org/licenses/by/4.0/), which permits unrestricted use, distribution, and reproduction in any medium, provided you give appropriate credit to the original author(s) and the source, provide a link to the Creative Commons license, and indicate if changes were made. 
gated by some authors (e.g. $[3,10,11,19])$. Thus, we will simply denote $A_{\mu}(\Omega)=A_{\mu}\left(\mathbb{R}^{N}\right)=$ $A_{\mu}$.

In this paper, for $\sum_{i=1}^{k} \mu_{i} \in[0, \bar{\mu})$, we use $H_{0}^{2}(\Omega)$ to denote the completion of $C_{0}^{\infty}(\Omega)$ with respect to the norm

$$
\|u\|:=\left(\int_{\Omega}\left(|\Delta u|^{2}-\sum_{i=1}^{k} \frac{\mu_{i} u^{2}}{\left|x-a_{i}\right|^{4}}\right) d x\right)^{\frac{1}{2}} .
$$

By (1.1), this norm is equivalent to the usual norm $\left(\int_{\Omega}|\Delta u|^{2} d x\right)^{\frac{1}{2}}$.

It is easily to see that Eq. $\left(E_{\lambda}\right)$ is variational and its solutions are critical points of the functional defined in $H_{0}^{2}(\Omega)$ by

$$
J_{\lambda}(u):=\frac{1}{2}\|u\|^{2}-\frac{1}{2^{*}} \int_{\Omega}|u|^{2^{*}}-\frac{\lambda}{q} \int_{\Omega}|u|^{q}, \quad u \in H_{0}^{2}(\Omega) .
$$

Then $J_{\lambda} \in C^{1}\left(H_{0}^{2}(\Omega), \mathbb{R}\right)$ and that

$$
\left\langle J_{\lambda}^{\prime}(u), v\right\rangle=\int_{\Omega}\left(\Delta u \Delta v-\sum_{i=1}^{k} \frac{\mu_{i} u v}{\left|x-a_{i}\right|^{4}}\right)-\int_{\Omega}|u|^{2^{*}-2} u v-\lambda \int_{\Omega}|u|^{q-2} u v, \quad \forall v \in H_{0}^{2}(\Omega) .
$$

In recent years problems related with the inequality (1.1) and the equations with biharmonic operator have been investigated in several works; we quote $[1,3,6-10,13,18,19]$. On the other hand, the biharmonic problems involving a Rellich-type potential and a critical Sobolev exponent have seldom been studied; we only find some results in $[10,18,19]$. Thus it is necessary for us to investigate the related biharmonic problems deeply. Very recently, Hsu and Zhang [16] studied the existence and multiplicity of nontrivial solution for the following equation:

$$
\begin{cases}\Delta^{2} u-\frac{\mu}{|x|^{4}} u=\frac{|u|^{*}(s)-2}{|x|^{5}} u+\lambda \frac{|u|^{q-2}}{|x|^{t}} u, & x \in \Omega, \\ u=\frac{\partial u}{\partial n}=0, & x \in \partial \Omega,\end{cases}
$$

where $\Omega \subset \mathbb{R}^{N}(N \geq 5)$ is a smooth bounded domain such that $0 \in \Omega, 0 \leq \mu<\bar{\mu}, 0 \leq s$, $t<4,1 \leq q<2, \lambda>0$.

In this paper, we study a biharmonic equation involving multiple Rellich-type potentials and a critical Sobolev exponent. It should be mentioned that the main technical difficulty to study equations like Eq. $\left(E_{\lambda}\right)$ is the lack of knowledge of the explicit form minimizers to the best Rellich-Sobolev constant $A_{\mu_{i}}$. However, as in [10] and [19], this difficulty can be overcome since the unique tool which is necessary to perform the needed asymptotic expansions is the asymptotic behavior at the origin and infinity of Rellich-Sobolev extremals and their first derivatives, which is established in Theorem 1.1 of [19]. We are only aware of the work in [18] which studied the existence and nonexistence of ground state solution to Eq. $\left(E_{\lambda}\right)$ when $\Omega=\mathbb{R}^{N}, k \geq 2$ and $\lambda=0$. Furthermore, Eq. $\left(E_{\lambda}\right)$ have never been studied when $\Omega$ is a smooth bounded domain and $k \geq 2$, and our results are new.

For $0 \leq \mu_{i}<\bar{\mu}$ and $a_{i} \in \Omega, i=1,2, \ldots, k$, we can define the constant:

$$
A_{\mu_{i}}:=\inf _{u \in H_{0}^{2}(\Omega) \backslash\{0\}} \frac{\int_{\Omega}\left(|\Delta u|^{2}-\mu_{i} \frac{u^{2}}{\left|x-a_{i}\right|^{4}}\right) d x}{\left(\int_{\Omega}|u|^{2^{*}} d x\right)^{\frac{2}{2^{*}}}} .
$$


The authors in $[10,19]$ proved that $A_{\mu_{i}}$ is attained in $\mathbb{R}^{N}$ by the functions

$$
\left\{y_{\varepsilon}^{\mu_{i}}\left(x-a_{i}\right)=\varepsilon^{\frac{4-N}{2}} U_{\mu_{i}}\left(\varepsilon^{-1}\left(x-a_{i}\right)\right), \varepsilon>0\right\},
$$

where $U_{\mu_{i}}(x)$ is positive, radially symmetric, radially decreasing, and solves

$$
\Delta^{2} u-\mu_{i} \frac{u}{|x|^{4}}=|u|^{2^{*}-1}, \quad x \in \mathbb{R}^{N} \backslash\{0\}, u>0
$$

which satisfies

$$
\int_{\mathbb{R}^{N}}\left(\left|\Delta y_{\varepsilon}^{\mu_{i}}\left(x-a_{i}\right)\right|^{2}-\mu_{i} \frac{\left|y_{\varepsilon}^{\mu_{i}}\left(x-a_{i}\right)\right|^{2}}{\left|x-a_{i}\right|^{4}}\right) d x=\int_{\mathbb{R}^{N}}\left|y_{\varepsilon}^{\mu_{i}}\left(x-a_{i}\right)\right|^{2^{*}} d x=A_{\mu_{i}}^{\frac{N}{4}} .
$$

Moreover, by setting $\rho=|x|$,

$$
\begin{aligned}
& U_{\mu}(\rho)=O_{1}\left(\rho^{-a(\mu)}\right), \quad \text { as } \rho \rightarrow 0, \\
& U_{\mu}(\rho)=O_{1}\left(\rho^{-b(\mu)}\right), \quad U_{\mu}^{\prime}(\rho)=O_{1}\left(\rho^{-b(\mu)-1}\right), \quad \text { as } \rho \rightarrow+\infty,
\end{aligned}
$$

where $a(\mu):=\frac{N-4}{2} f(\mu), b(\mu):=\frac{N-4}{2}(2-f(\mu))$ and $f:[0, \bar{\mu}] \rightarrow[0,1]$ is defined as

$$
f(\mu):=1-\frac{\sqrt{N^{2}-4 N+8-4 \sqrt{(N-2)^{2}+\mu}}}{N-4}, \quad \mu \in[0, \bar{\mu}] .
$$

From Lemma 2.1 in [18], it follows that for $\mu \in[0, \bar{\mu})$

$$
0 \leq a(\mu) \leq \delta \leq b(\mu) \leq 2 \delta, \quad \delta:=\frac{N-4}{2}
$$

Furthermore, there exist positive constants $\mathcal{C}_{1}(\mu)$ and $\mathcal{C}_{2}(\mu)$ such that

$$
0<\mathcal{C}_{1}(\mu) \leq \mathcal{U}_{\mu}(x)\left(|x|^{\frac{a(\mu)}{\delta}}+|x|^{\frac{b(\mu)}{\delta}}\right)^{\delta} \leq \mathcal{C}_{2}(\mu), \quad \forall x \in \mathbb{R}^{N} \backslash\{0\}
$$

Without loss of generality, throughout this paper we assume that

$(\mathcal{H}) 0 \leq \mu_{1} \leq \mu_{2} \leq \cdots \leq \mu_{k}<\bar{\mu}, \sum_{i=1}^{k} \mu_{i}<\bar{\mu}$, and $2^{*}:=\frac{2 N}{N-4}$.

In this paper, we define the following constants and notations:

$$
\|u\|^{2}=\int_{\Omega}\left(|\Delta u|^{2}-\sum_{i=1}^{k} \frac{\mu_{i} u^{2}}{\left|x-a_{i}\right|^{4}}\right) d x \text { is the norm in } H_{0}^{2}(\Omega) ;
$$

$H^{-2}(\Omega)$ : the dual space of $H_{0}^{2}(\Omega)$;

$\langle\cdot, \cdot\rangle$ : the usual scalar product in $H_{0}^{2}(\Omega)$;

$$
\begin{aligned}
& B_{r}(a)=\{x:|x-a|<r\}, \quad \overline{B_{r}(a)}=\{x:|x-a| \leq r\}, \quad a \in \mathbb{R}^{\mathbb{N}}, \quad r>0 ; \\
& \mu^{*}:=\frac{1}{16}\left(N^{2}-16\right)\left(N^{2}-8 N\right), \quad N \geq 9 ; \\
& S=\inf _{u \in H_{0}^{2}(\Omega) \backslash\{0\}} \frac{\int_{\Omega}\left(|\Delta u|^{2}-\sum_{i=1}^{k} \mu_{i} \frac{u^{2}}{\left|x-a_{i}\right|^{4}}\right) d x}{\left(\int_{\Omega}|u|^{2^{*}} d x\right)^{\frac{2}{2^{*}}}} ;
\end{aligned}
$$




$$
\begin{aligned}
& \Lambda_{0}:=\left(\frac{2-q}{2^{*}-q}\right)^{\frac{2-q}{2^{*}-2}}\left(\frac{2^{*}-2}{2^{*}-q}\right)|\Omega|^{-\frac{2^{*}-q}{2^{*}}} S^{\frac{(2-q) N}{8}+\frac{q}{2}} ; \\
& \lambda_{1}:=\inf _{u \in H_{0}^{2}(\Omega) \backslash\{0\}} \frac{\int_{\Omega}\left(|\Delta u|^{2}-\sum_{i=1}^{k} \mu_{i} \frac{u^{2}}{\left|x-a_{i}\right|^{\mid}}\right) d x}{\int_{\Omega}|u|^{2} d x} .
\end{aligned}
$$

Since the embedding $H_{0}^{2}(\Omega) \hookrightarrow L^{2}(\Omega)$ is compact, by choosing a minimizing sequence, we easily infer that $\lambda_{1}$ can be obtained in $H_{0}^{2}(\Omega)$, and $\lambda_{1}>0 . C, C_{1}, C_{2}, \ldots$ denote various positive constants. For all $\varepsilon>0, \tau>0, O\left(\varepsilon^{\tau}\right)$ denotes the quantity satisfying $\left|O\left(\varepsilon^{\tau}\right) / \varepsilon^{\tau}\right| \leq C$ and $o\left(\varepsilon^{\tau}\right)$ means $\left|o\left(\varepsilon^{\tau}\right) / \varepsilon^{\tau}\right| \rightarrow 0$ as $\varepsilon \rightarrow \varepsilon_{0}, o_{n}(1)$ denotes $o_{n}(1) \rightarrow 0$ as $n \rightarrow \infty$ and $O_{1}\left(\varepsilon^{\tau}\right)$ $\left(\varepsilon \rightarrow \varepsilon_{0}\right)$ means that there exist the constants $C_{1}, C_{2}>0$ such that $C_{1} \varepsilon^{\tau} \leq O_{1}\left(\varepsilon^{\tau}\right) \leq C_{2} \varepsilon^{\tau}$ as $\varepsilon \rightarrow \varepsilon_{0} .|\Omega|$ denotes the Lebesgue measure of $\Omega$ and omit $d x$ in integrals for convenience.

Let $1 \leq q<2^{*}$, by the Hölder inequality and (1.4), for all $u \in H_{0}^{2}(\Omega)$, we obtain

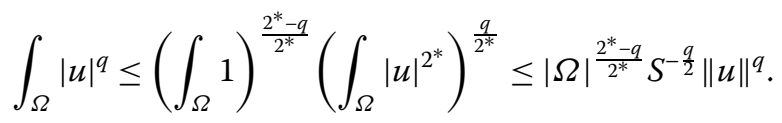

We are now ready to state our main results.

Theorem 1.1 Let $N \geq 5,1 \leq q<2$ and assume that $(\mathcal{H})$ holds, then we have the following results.

(i) If $\lambda \in\left(0, \Lambda_{0}\right)$, then $E q$. $\left(E_{\lambda}\right)$ has at least one nontrivial solution.

(ii) If $\lambda \in\left(0, \frac{q}{2} \Lambda_{0}\right)$, then $E q$. $\left(E_{\lambda}\right)$ has at least two nontrivial solutions.

Theorem 1.2 Let $N \geq 5,2 \leq q<2^{*}$ and assume that $(\mathcal{H})$ and one of the following conditions holds:

(i) $\lambda>0, \bar{q}<q<2^{*}$, where

$$
\bar{q}=\max \left\{2, \frac{N}{b\left(\mu_{k}\right)}, \frac{4\left(N-2-b\left(\mu_{k}\right)\right)}{N-4}\right\} .
$$

(ii) $N \geq 8,0<\lambda<\lambda_{1}, q=2,0 \leq \mu_{k} \leq \mu^{*}$.

Then Eq. $\left(E_{\lambda}\right)$ has at least one nontrivial solution.

This paper is organized as follows. In Sect. 2, we give some properties of Nehari manifold. In Sects. 3 and 4, we prove Theorem 1.1. In Sect. 5, we prove Theorem 1.2.

\section{Nehari manifold}

In this section, we will give some properties of Nehari manifold. As the energy functional $J_{\lambda}$ is not bounded below on $H_{0}^{2}(\Omega)$, it is useful to consider the functional on the Nehari manifold

$$
\mathcal{M}_{\lambda}=\left\{u \in H_{0}^{2}(\Omega) \backslash\{0\}:\left\langle J_{\lambda}^{\prime}(u), u\right\rangle=0\right\} .
$$

Thus, $u \in \mathcal{M}_{\lambda}$ if and only if

$$
\left\langle J_{\lambda}^{\prime}(u), u\right\rangle=\|u\|^{2}-\int_{\Omega}|u|^{2^{*}}-\lambda \int_{\Omega}|u|^{q}=0 .
$$


Note that $\mathcal{M}_{\lambda}$ contains every nonzero solution of Eq. $\left(E_{\lambda}\right)$. Moreover, we have the following results.

Lemma 2.1 Let $N \geq 5,1 \leq q<2$ and $\lambda \in\left(0, \Lambda_{0}\right)$ where $\Lambda_{0}$ is the same as in (1.5). Then $J_{\lambda}$ is coercive and bounded below on $\mathcal{M}_{\lambda}$.

Proof If $u \in \mathcal{M}_{\lambda}$, then by (1.4), (2.1), and the Hölder inequality

$$
\begin{aligned}
J_{\lambda}(u) & =\frac{1}{2}\|u\|^{2}+\frac{1}{2^{*}}\left(\lambda \int_{\Omega}|u|^{q}-\|u\|^{2}\right)-\frac{\lambda}{q} \int_{\Omega}|u|^{q} \\
& =\frac{2^{*}-2}{22^{*}}\|u\|^{2}-\lambda\left(\frac{2^{*}-q}{2^{*} q}\right) \int_{\Omega}|u|^{q} \\
& \geq \frac{2}{N}\|u\|^{2}-\lambda\left(\frac{2^{*}-q}{2^{*} q}\right)|\Omega|^{\frac{2^{*}-q}{2^{*}}} S^{-\frac{q}{2}}\|u\|^{q} .
\end{aligned}
$$

Thus, $J_{\lambda}$ is coercive and bounded below on $\mathcal{M}_{\lambda}$.

Define $\psi_{\lambda}: H_{0}^{2}(\Omega) \rightarrow \mathbb{R}$, by $\psi_{\lambda}(u)=\left\langle J_{\lambda}^{\prime}(u), u\right\rangle$, that is,

$$
\psi_{\lambda}(u)=\|u\|^{2}-\int_{\Omega}|u|^{2^{*}}-\lambda \int_{\Omega}|u|^{q} .
$$

Then we see that $\psi_{\lambda} \in C^{1}\left(H_{0}^{2}(\Omega), \mathbb{R}\right), \mathcal{M}_{\lambda}=\psi_{\lambda}^{-1}(0) \backslash\{0\}$, and for all $u \in \mathcal{M}_{\lambda}$,

$$
\begin{aligned}
\left\langle\psi_{\lambda}^{\prime}(u), u\right\rangle & =2\|u\|^{2}-2^{*} \int_{\Omega}|u|^{2^{*}}-\lambda q \int_{\Omega}|u|^{q} \\
& =(2-q)\|u\|^{2}-\left(2^{*}-q\right) \int_{\Omega}|u|^{2^{*}} \\
& =\left(2-2^{*}\right)\|u\|^{2}-\lambda\left(q-2^{*}\right) \int_{\Omega}|u|^{q} .
\end{aligned}
$$

We split $\mathcal{M}_{\lambda}$ into three parts:

$$
\begin{aligned}
& \mathcal{M}_{\lambda}^{+}=\left\{u \in \mathcal{M}_{\lambda}:\left\langle\psi_{\lambda}^{\prime}(u), u\right\rangle>0\right\}, \\
& \mathcal{M}_{\lambda}^{0}=\left\{u \in \mathcal{M}_{\lambda}:\left\langle\psi_{\lambda}^{\prime}(u), u\right\rangle=0\right\}, \\
& \mathcal{M}_{\lambda}^{-}=\left\{u \in \mathcal{M}_{\lambda}:\left\langle\psi_{\lambda}^{\prime}(u), u\right\rangle<0\right\} .
\end{aligned}
$$

We now derive some basic properties of $\mathcal{M}_{\lambda}^{+}, \mathcal{M}_{\lambda}^{0}$ and $\mathcal{M}_{\lambda}^{-}$.

Lemma 2.2 Assume that $u_{0}$ is a local minimizer for $J_{\lambda}$ on $\mathcal{M}_{\lambda}$ and $u_{0} \notin \mathcal{M}_{\lambda}^{0}$. Then $J_{\lambda}^{\prime}\left(u_{0}\right)=$ 0 in $H^{-2}(\Omega)$.

Proof See [5, Theorem 2.3].

Moreover, we have the following result.

Lemma 2.3 If $\lambda \in\left(0, \Lambda_{0}\right)$, then $\mathcal{M}_{\lambda}^{0}=\emptyset$. 
Proof Arguing by contradiction, we assume that there exists a $\lambda \in\left(0, \Lambda_{0}\right)$ such that $\mathcal{M}_{\lambda}^{0} \neq$ $\emptyset$. Then, for $u \in \mathcal{M}_{\lambda}^{0}$ by (1.4) and (2.4), we have

$$
\frac{2-q}{2^{*}-q}\|u\|^{2}=\int_{\Omega}|u|^{2^{*}} \leq S^{-\frac{2^{*}}{2}}\|u\|^{2^{*}}
$$

and so

$$
\|u\| \geq\left(\frac{2-q}{2^{*}-q}\right)^{\frac{1}{2^{*}-2}} S^{\frac{2^{*}}{2\left(2^{*}-2\right)}} .
$$

Similarly, using (1.7), (2.5), and the Hölder inequality, we have

$$
\|u\|^{2}=\lambda \frac{2^{*}-q}{2^{*}-2} \int_{\Omega}|u|^{q} \leq \lambda \frac{2^{*}-q}{2^{*}-2}|\Omega|^{\frac{2^{*}-q}{2^{*}}} S^{-\frac{q}{2}}\|u\|^{q}
$$

which implies

$$
\|u\| \leq\left[\lambda \frac{2^{*}-q}{2^{*}-2}|\Omega|^{\frac{2^{*}-q}{2^{*}}} S^{-\frac{q}{2}}\right]^{\frac{1}{2-q}}
$$

Hence, we must have

$$
\lambda \geq\left(\frac{2-q}{2^{*}-q}\right)^{\frac{2-q}{2^{*}-2}}\left(\frac{2^{*}-2}{2^{*}-q}\right)|\Omega|^{-\frac{2^{*}-q}{2^{*}}} S^{\frac{(2-q) N}{8}+\frac{q}{2}}=\Lambda_{0},
$$

which is a contradiction. This completes the proof.

For each $u \in H_{0}^{2}(\Omega) \backslash\{0\}$, let

$$
\tau_{\max }=\left(\frac{(2-q)\|u\|^{2}}{\left(2^{*}-q\right) \int_{\Omega}|u|^{2^{*}}}\right)^{\frac{1}{2^{*}-2}}>0 .
$$

Similar to Lemma 2.7 in [14], we can get the following result.

Lemma 2.4 If $\lambda \in\left(0, \Lambda_{0}\right)$, then, for each $u \in H_{0}^{2}(\Omega) \backslash\{0\}$, the set $\{\tau u: \tau>0\}$ intersects $\mathcal{M}_{\lambda}$ exactly twice. More specifically, there exist a unique $\tau^{-}=\tau^{-}(u)>0$ such that $\tau^{-} u \in \mathcal{M}_{\lambda}^{-}$ and a unique $\tau^{+}=\tau^{+}(u)>0$ such that $\tau^{+} u \in \mathcal{M}_{\lambda}^{+}$. Moreover, $\tau^{+}<\tau_{\max }<\tau^{-}$and

$$
J_{\lambda}\left(\tau^{+} u\right)=\inf _{0 \leq \tau \leq \tau_{\max }} J_{\lambda}(\tau u), \quad J_{\lambda}\left(\tau^{-} u\right)=\sup _{\tau \geq \tau_{\max }} J_{\lambda}(\tau u)
$$

Proof The proof is similar to that of [14, Lemma 2.7] and is omitted.

\section{Existence of ground state solutions in the case of $1 \leq q<2$}

First, we remark that it follows from Lemma 2.3 that

$$
\mathcal{M}_{\lambda}=\mathcal{M}_{\lambda}^{+} \cup \mathcal{M}_{\lambda}^{-}
$$


for all $\lambda \in\left(0, \Lambda_{0}\right)$. Furthermore, by Lemma 2.4 it follows that $\mathcal{M}_{\lambda}^{+}$and $\mathcal{M}_{\lambda}^{-}$are non-empty and by Lemma 2.1 we may define

$$
\alpha_{\lambda}=\inf _{u \in \mathcal{M}_{\lambda}} J_{\lambda}(u) ; \quad \alpha_{\lambda}^{+}=\inf _{u \in \mathcal{M}_{\lambda}^{+}} J_{\lambda}(u) ; \quad \alpha_{\lambda}^{-}=\inf _{u \in \mathcal{M}_{\lambda}^{-}} J_{\lambda}(u)
$$

\section{Lemma 3.1 The following facts hold.}

(i) If $\lambda \in\left(0, \Lambda_{0}\right)$, then $\alpha_{\lambda} \leq \alpha_{\lambda}^{+}<0$.

(ii) If $\lambda \in\left(0, \frac{q}{2} \Lambda_{0}\right)$, then $\alpha_{\lambda}^{-}>c_{0}$ for some $c_{0}>0$.

In particular, for each $\lambda \in\left(0, \frac{q}{2} \Lambda_{0}\right)$, we have $\alpha_{\lambda}^{+}=\alpha_{\lambda}$.

Proof (i) Let $u \in \mathcal{M}_{\lambda}^{+}$. By (2.4)

$$
\frac{2-q}{2^{*}-q}\|u\|^{2}>\int_{\Omega}|u|^{2^{*}}
$$

and so

$$
\begin{aligned}
J_{\lambda}(u) & =\left(\frac{1}{2}-\frac{1}{q}\right)\|u\|^{2}+\left(\frac{1}{q}-\frac{1}{2^{*}}\right) \int_{\Omega}|u|^{2^{*}} \\
& <\left[\left(\frac{1}{2}-\frac{1}{q}\right)+\left(\frac{1}{q}-\frac{1}{2^{*}}\right)\left(\frac{2-q}{2^{*}-q}\right)\right]\|u\|^{2} \\
& =-\frac{\left(2^{*}-2\right)(2-q)}{22^{*} q}\|u\|^{2}<0 .
\end{aligned}
$$

Therefore, from the definition of $\alpha_{\lambda}$ and $\alpha_{\lambda}^{+}$, we can deduce that $\alpha_{\lambda} \leq \alpha_{\lambda}^{+}<0$.

(ii) Let $u \in \mathcal{M}_{\lambda}^{-}$. By (2.4)

$$
\frac{2-q}{2^{*}-q}\|u\|^{2}<\int_{\Omega}|u|^{2^{*}}
$$

Moreover, by (1.4) we have

$$
\int_{\Omega}|u|^{2^{*}} \leq S^{-\frac{2^{*}}{2}}\|u\|^{2^{*}}
$$

This implies

$$
\|u\|>\left(\frac{2-q}{2^{*}-q}\right)^{\frac{1}{2^{*}-2}} S^{\frac{N}{8}} \quad \text { for all } u \in \mathcal{M}_{\lambda}^{-} .
$$

By (2.3) and (3.1), we have

$$
\begin{aligned}
J_{\lambda}(u) & \geq\|u\|^{q}\left[\frac{2}{N}\|u\|^{2-q}-\lambda\left(\frac{2^{*}-q}{2^{*} q}\right)|\Omega|^{\frac{2^{*}-q}{2^{*}}} S^{-\frac{q}{2}}\right] \\
& >\left(\frac{2-q}{2^{*}-q}\right)^{\frac{q}{2^{*}-2}} S^{\frac{q N}{8}}\left[\frac{2}{N}\left(\frac{2-q}{2^{*}-q}\right)^{\frac{2-q}{2^{*}-2}} S^{\frac{(2-q) N}{8}}-\lambda\left(\frac{2^{*}-q}{2^{*} q}\right)|\Omega|^{\frac{2^{*}-q}{2^{*}}} S^{-\frac{q}{2}}\right] \\
& =\left(\frac{q}{2} \Lambda_{0}-\lambda\right)\left(\frac{2-q}{2^{*}-q}\right)^{\frac{q}{2^{*}-2}}\left(\frac{2^{*}-q}{2^{*} q}\right)|\Omega|^{\frac{2^{*}-q}{2^{*}}} S^{\frac{(N-4) q}{8}} .
\end{aligned}
$$


Thus, if $\lambda \in\left(0, \frac{q}{2} \Lambda_{0}\right)$, then there exists $c_{0}>0$ such that

$$
J_{\lambda}(u)>c_{0} \quad \text { for all } u \in \mathcal{M}_{\lambda}^{-} .
$$

Consequently, this completes the proof.

\section{Remark 3.2}

(i) If $\lambda \in\left(0, \Lambda_{0}\right)$, then, by (1.7), (2.5), and the Hölder inequality, for each $u \in \mathcal{M}_{\lambda}^{+}$we have

$$
\begin{aligned}
\|u\|^{2} & <\lambda \frac{2^{*}-q}{2^{*}-2} \int_{\Omega}|u|^{q} \\
& \leq \lambda \frac{2^{*}-q}{2^{*}-2}|\Omega|^{\frac{2^{*}-q}{2^{*}}} S^{-\frac{q}{2}}\|u\|^{q}
\end{aligned}
$$

and so

$$
\|u\|<\left[\lambda \frac{2^{*}-q}{2^{*}-2}|\Omega|^{\frac{2^{*}-q}{2^{*}}} S^{-\frac{q}{2}}\right]^{\frac{1}{2-q}} \quad \text { for all } u \in \mathcal{M}_{\lambda}^{+} .
$$

(ii) If $\lambda \in\left(0, \frac{q}{2} \Lambda_{0}\right)$, then, by Lemma 2.4 and Lemma 3.1(ii), for each $u \in \mathcal{M}_{\lambda}^{-}$we have

$$
J_{\lambda}(u)=\sup _{t \geq 0} J_{\lambda}(t u)
$$

We define the Palais-Smale (indicated simply by the prefix "(PS)-") sequences, (PS)values, and (PS)-conditions in $H_{0}^{2}(\Omega)$ for $J_{\lambda}$ as follows.

\section{Definition 3.3}

(i) For $c \in \mathbb{R}$, a sequence $\left\{u_{n}\right\}$ is a (PS) $c_{c}$-sequence in $H_{0}^{2}(\Omega)$ for $J_{\lambda}$ if $J_{\lambda}\left(u_{n}\right)=c+o_{n}(1)$ and $J_{\lambda}^{\prime}\left(u_{n}\right)=o_{n}(1)$ strongly in $H^{-2}(\Omega)$ as $n \rightarrow \infty$.

(ii) $c \in \mathbb{R}$ is a (PS)-value in $H_{0}^{2}(\Omega)$ for $J_{\lambda}$ if there exists a (PS) $)_{c}$-sequence in $H_{0}^{2}(\Omega)$ for $J_{\lambda}$.

(iii) $J_{\lambda}$ satisfies the (PS) $c_{c}$-condition in $H_{0}^{2}(\Omega)$ if any $(\mathrm{PS})_{c}$-sequence $\left\{u_{n}\right\}$ in $H_{0}^{2}(\Omega)$ for $J_{\lambda}$ contains a convergent subsequence.

Now, we use the Ekeland variational principle [12] to get the following results.

\section{Proposition 3.4}

(i) If $\lambda \in\left(0, \Lambda_{0}\right)$, then there exists a (PS) $\alpha_{\lambda}$-sequence $\left\{u_{n}\right\} \subset \mathcal{M}_{\lambda}$ in $H_{0}^{2}(\Omega)$ for $J_{\lambda}$.

(ii) If $\lambda \in\left(0, \frac{q}{2} \Lambda_{0}\right)$, then there exists a (PS) $\alpha_{\lambda}^{-}$-sequence $\left\{u_{n}\right\} \subset \mathcal{M}_{\lambda}^{-}$in $H_{0}^{2}(\Omega)$ for $J_{\lambda}$.

Proof The proof is similar to that of [14, Proposition 3.3] and is omitted.

Now, we establish the existence of a local minimum for $J_{\lambda}$ on $\mathcal{M}_{\lambda}$.

Theorem 3.5 Let $N \geq 5,1 \leq q<2$ and assume that the condition $(\mathcal{H})$ holds. If $\lambda \in\left(0, \Lambda_{0}\right)$, then $J_{\lambda}$ has a minimizer $u_{\lambda}$ in $\mathcal{M}_{\lambda}^{+}$and we have the following results.

(i) $J_{\lambda}\left(u_{\lambda}\right)=\alpha_{\lambda}=\alpha_{\lambda}^{+}$.

(ii) $u_{\lambda}$ is a nontrivial solution of Eq. $\left(E_{\lambda}\right)$.

(iii) $\left\|u_{\lambda}\right\| \rightarrow 0$ as $\lambda \rightarrow 0^{+}$. 
Proof By Proposition 3.4(i), there is a minimizing sequence $\left\{u_{n}\right\}$ for $J_{\lambda}$ on $\mathcal{M}_{\lambda}$ such that

$$
J_{\lambda}\left(u_{n}\right)=\alpha_{\lambda}+o_{n}(1) \text { and } J_{\lambda}^{\prime}\left(u_{n}\right)=o_{n}(1) \text { in } H^{-2}(\Omega) .
$$

Since $J_{\lambda}$ is coercive on $\mathcal{M}_{\lambda}$ (see Lemma 2.1), we see that $\left\{u_{n}\right\}$ is bounded in $H_{0}^{2}(\Omega)$. Thus, passing a subsequence if necessary, there exists $u_{\lambda} \in H_{0}^{2}(\Omega)$ such that as $n \rightarrow \infty$

$$
\begin{cases}u_{n} \rightarrow u_{\lambda} & \text { weakly in } H_{0}^{2}(\Omega), \\ u_{n} \rightarrow u_{\lambda} & \text { strongly in } L^{q}(\Omega) \text { for } 1 \leq q<2^{*} \\ u_{n} \rightarrow u_{\lambda} & \text { almost everywhere in } \Omega\end{cases}
$$

It follows that

$$
\lambda \int_{\Omega}\left|u_{n}\right|^{q} \rightarrow \lambda \int_{\Omega}\left|u_{\lambda}\right|^{q} \quad \text { as } n \rightarrow \infty, \forall 1 \leq q<2
$$

By (3.3), (3.4) and (3.5), it is easy to see that $u_{\lambda}$ is a weak solution of Eq. $\left(E_{\lambda}\right)$. From $\left\{u_{n}\right\} \subset \mathcal{M}_{\lambda},(2.2)$ and (3.5), we deduce that

$$
\begin{aligned}
J_{\lambda}\left(u_{n}\right) & =\frac{2^{*}-2}{22^{*}}\left\|u_{n}\right\|^{2}-\lambda\left(\frac{2^{*}-q}{2^{*} q}\right) \int_{\Omega}\left|u_{n}\right|^{q} \\
& \geq-\lambda\left(\frac{2^{*}-q}{2^{*} q}\right) \int_{\Omega}\left|u_{n}\right|^{q} \\
& \rightarrow-\lambda\left(\frac{2^{*}-q}{2^{*} q}\right) \int_{\Omega}\left|u_{\lambda}\right|^{q} .
\end{aligned}
$$

This and $J_{\lambda}\left(u_{n}\right) \rightarrow \alpha_{\lambda}<0$ (see Lemma 3.1(i)) yield $\int_{\Omega}\left|u_{\lambda}\right|^{q}>0$, that is, $u_{\lambda} \not \equiv 0$. We use $J_{\lambda}\left(u_{\lambda}\right)=J_{\lambda}\left(\left|u_{\lambda}\right|\right)$ and $\left|u_{\lambda}\right| \in \mathcal{M}_{\lambda}$. Thus by Lemma 2.2, we may assume that $u_{\lambda}$ is a nontrivial nonnegative solution of Eq. $\left(E_{\lambda}\right)$.

Now we prove that up to a subsequence, $u_{n} \rightarrow u_{\lambda}$ strongly in $H_{0}^{2}(\Omega)$ and $J_{\lambda}\left(u_{\lambda}\right)=\alpha_{\lambda}$. From the fact $u_{n}, u \in \mathcal{M}_{\lambda}$ and Fatou's lemma, we have

$$
\begin{aligned}
\alpha_{\lambda} & \leq J_{\lambda}\left(u_{\lambda}\right)=\frac{2^{*}-2}{22^{*}}\left\|u_{\lambda}\right\|^{2}-\lambda\left(\frac{2^{*}-q}{2^{*} q}\right) \int_{\Omega}\left|u_{\lambda}\right|^{q} \\
& \leq \liminf _{n \rightarrow \infty}\left[\frac{2^{*}-2}{22^{*}}\left\|u_{n}\right\|^{2}-\lambda\left(\frac{2^{*}-q}{2^{*} q}\right) \int_{\Omega}\left|u_{n}\right|^{q}\right] \\
& =\liminf _{n \rightarrow \infty} J_{\lambda}\left(u_{n}\right) \\
& =\alpha_{\lambda},
\end{aligned}
$$

which implies that $J_{\lambda}\left(u_{\lambda}\right)=\alpha_{\lambda}$ and $\lim _{n \rightarrow \infty}\left\|u_{n}\right\|^{2}=\left\|u_{\lambda}\right\|^{2}$. Standard argument shows that $u_{n} \rightarrow u_{\lambda}$ strongly in $H_{0}^{2}(\Omega)$.

Next, we claim $u_{\lambda} \in \mathcal{M}_{\lambda}^{+}$. Indeed, if $u_{\lambda} \in \mathcal{M}_{\lambda}^{-}$, by Lemma 2.4 , there exist unique $\tau_{\lambda}^{+}$and $\tau_{\lambda}^{-}$such that $\tau_{\lambda}^{+} u_{\lambda} \in \mathcal{M}_{\lambda}^{+}, \tau_{\lambda}^{-} u_{\lambda} \in \mathcal{M}_{\lambda}^{-}$and $\tau_{\lambda}^{+}<\tau_{\lambda}^{-}=1$. Since

$$
\frac{d}{d \tau} J_{\lambda}\left(\tau_{\lambda}^{+} u_{\lambda}\right)=0 \quad \text { and } \quad \frac{d^{2}}{d \tau^{2}} J_{\lambda}\left(\tau_{\lambda}^{+} u_{\lambda}\right)>0
$$


there exists $\bar{\tau} \in\left(\tau_{\lambda}^{+}, \tau_{\lambda}^{-}\right)$such that $J_{\lambda}\left(\tau_{\lambda}^{+} u_{\lambda}\right)<J_{\lambda}\left(\bar{\tau} u_{\lambda}\right)$. By Lemma 2.4 we get

$$
J_{\lambda}\left(\tau_{\lambda}^{+} u_{\lambda}\right)<J_{\lambda}\left(\bar{\tau} u_{\lambda}\right) \leq J_{\lambda}\left(\tau_{\lambda}^{-} u_{\lambda}\right)=J_{\lambda}\left(u_{\lambda}\right),
$$

which contradicts $J_{\lambda}\left(u_{\lambda}\right)=\alpha_{\lambda}$. Consequently, $u_{\lambda} \in \mathcal{M}_{\lambda}^{+}$.

Finally, by $u_{\lambda} \in \mathcal{M}_{\lambda}^{+}$and (3.2), we obtain

$$
\left\|u_{\lambda}\right\|<\left[\lambda \frac{2^{*}-q}{2^{*}-2}|\Omega|^{\frac{2^{*}-q}{2^{*}}} S^{-\frac{q}{2}}\right]^{\frac{1}{2-q}} \text { for all } u \in \mathcal{M}_{\lambda}^{+}
$$

This implies that $\left\|u_{\lambda}\right\| \rightarrow 0$ as $\lambda \rightarrow 0^{+}$, and completes the proof.

\section{Multiplicity of nontrivial solutions in the case of $1 \leq q<2$}

In this section, we will establish the existence of the second nontrivial solution of Eq. $\left(E_{\lambda}\right)$ by proving that $J_{\lambda}$ attains a local minimum on $\mathcal{M}_{\lambda}^{-}$.

Lemma 4.1 If $\left\{u_{n}\right\} \subset H_{0}^{2}(\Omega)$ is a (PS) $)_{c}$-sequence for $J_{\lambda}$, then $\left\{u_{n}\right\}$ is bounded in $H_{0}^{2}(\Omega)$.

Proof The proof is similar to that of [15, Lemma 4.1] and is omitted.

We recall that

$$
A_{\mu_{i}}:=\inf _{u \in H_{0}^{2}(\Omega) \backslash\{0\}} \frac{\int_{\Omega}\left(|\Delta u|^{2}-\mu_{i} \frac{u^{2}}{\left|x-a_{i}\right|^{4}}\right) d x}{\left(\int_{\Omega}|u|^{2^{*}} d x\right)^{\frac{2}{2^{*}}}} .
$$

Lemma 4.2 Let $N \geq 5,1 \leq q<2$ and assume that $(\mathcal{H})$ holds. If $\left\{u_{n}\right\} \subset H_{0}^{2}(\Omega)$ is a (PS) $c^{-}$ sequence for $J_{\lambda}$ with $c \in\left(0, \frac{2}{N} A_{\mu_{k}}^{\frac{N}{4}}\right)$, then there exists a subsequence of $\left\{u_{n}\right\}$ converging weakly to a nonzero solution of $E q$. $\left(E_{\lambda}\right)$.

Proof Let $\left\{u_{n}\right\} \subset H_{0}^{2}(\Omega)$ be a (PS) $)_{c}$-sequence for $J_{\lambda}$ with $c \in\left(0, \frac{2}{N} A_{\mu_{k}}^{\frac{N}{4}}\right)$. We know from Lemma 4.1 that $\left\{u_{n}\right\}$ is bounded in $H_{0}^{2}(\Omega)$. Then there exists a subsequence of $\left\{u_{n}\right\}$ (still denoted by $\left.\left\{u_{n}\right\}\right)$ and $u_{0} \in H_{0}^{2}(\Omega)$ such that $u_{n} \rightarrow u_{0}$ in $H_{0}^{2}(\Omega), u_{n} \rightarrow u_{0}$ almost everywhere in $\Omega$, and $u_{n} \rightarrow u_{0}$ in $L^{q}(\Omega)$ for any $1 \leq q<2^{*}$ as $n \rightarrow \infty$. It is easy to see that $J_{\lambda}^{\prime}\left(u_{0}\right)=0$ and

$$
\lambda \int_{\Omega}\left|u_{n}\right|^{q}=\lambda \int_{\Omega}\left|u_{0}\right|^{q}+o_{n}(1)
$$

Next we verify that $u_{0} \not \equiv 0$. Arguing by contradiction, we assume $u_{0} \equiv 0$. By the concentration compactness principle (see $[20,21])$ there exists a subsequence, still denoted by $\left\{u_{n}\right\}$, an at most countable set $\mathcal{J}$, a set of different points $\left\{x_{j}\right\}_{j \in \mathcal{J}} \subset \Omega \backslash\left\{a_{1}, a_{2}, \ldots, a_{k}\right\}$, nonnegative real numbers $\widetilde{\mu_{x_{j}}}, \widetilde{v_{x_{j}}}, j \in \mathcal{J}$ and $\widetilde{\mu_{a_{i}}}, \widetilde{\gamma_{a_{i}}}, \widetilde{v_{a_{i}}}(1 \leq i \leq k)$ such that

$$
\left\{\begin{array}{l}
\left|\Delta u_{n}\right|^{2}-d \widetilde{\mu} \geq\left|\Delta u_{0}\right|^{2}+\sum_{j \in \mathcal{J}} \widetilde{\mu_{x_{j}}} \delta_{x_{j}}+\sum_{i=1}^{k} \widetilde{\mu_{a_{i}}} \delta_{a_{i}}, \\
\mu_{i} \frac{u_{n}^{2}}{\left|x-a_{i}\right|^{4}}-d \widetilde{\gamma_{a_{i}}}=\mu_{i} \frac{u_{0}^{2}}{\left|x-a_{i}\right|^{4}}+\widetilde{\gamma_{a_{i}}} \delta_{a_{i}}, \\
\left|u_{n}\right|^{2^{*}} \rightarrow d \widetilde{v}=\left|u_{0}\right|^{2}+\sum_{j \in \mathcal{J}} \widetilde{v_{x_{j}}} \delta_{x_{j}}+\sum_{i=1}^{k} \widetilde{v_{a_{i}}} \delta_{a_{i}},
\end{array}\right.
$$


where $\delta_{x}$ is the Dirac mass at $x$. By the Rellich inequalities, we get

$$
\widetilde{\mu_{a_{i}}}-\mu_{i} \widetilde{\gamma_{a_{i}}} \geq A_{\mu_{i}} \widetilde{v_{a_{i}}} \frac{2}{2^{*}}, \quad 1 \leq i \leq k
$$

Claim 1 . We claim that $\mathcal{J}$ is finite and for any $j \in \mathcal{J}$, either

$$
\tilde{v_{x_{j}}}=0 \quad \text { or } \quad \tilde{v_{x_{j}}} \geq A_{0}^{\frac{N}{4}} .
$$

In fact, let $\varepsilon>0$ be small enough such that $a_{i} \notin B_{2 \varepsilon}\left(x_{j}\right)$ for all $1 \leq i \leq k$ and $B_{2 \varepsilon}\left(x_{i}\right) \cap$ $B_{2 \varepsilon}\left(x_{j}\right)=\varnothing$ for $i \neq j, i, j \in \mathcal{J}$. Let $\phi_{\varepsilon}^{j}$ be a smooth cut-off function centered at $x_{j}$ such that $0 \leq \phi_{\varepsilon}^{j} \leq 1, \phi_{\varepsilon}^{j}=1$ for $\left|x-x_{j}\right| \leq \varepsilon, \phi_{\varepsilon}^{j}=0$ for $\left|x-x_{j}\right| \geq 2 \varepsilon,\left|\nabla \phi_{\varepsilon}^{j}\right| \leq \frac{2}{\varepsilon}$ and $\left|\Delta \phi_{\varepsilon}^{j}\right| \leq \frac{2}{\varepsilon^{2}}$. Consider the sequence $\left\{\phi_{\varepsilon}^{j} u_{n}\right\}$; it is obvious that this sequence is bounded in $H_{0}^{2}(\Omega)$. Then (4.1) implies

$$
\lim _{n \rightarrow \infty}\left\langle J_{\lambda}^{\prime}\left(u_{n}\right), \phi_{\varepsilon}^{j} u_{n}\right\rangle=0
$$

Moreover, by (4.2) we deduce

$$
\int_{\Omega} \sum_{i=1}^{k} \phi_{\varepsilon}^{j} d \widetilde{\gamma_{a_{i}}}+\int_{\Omega} \phi_{\varepsilon}^{j} d \widetilde{v}+\lambda \int_{\Omega}\left|u_{0}\right|^{q} \phi_{\varepsilon}^{j} d x=\lim _{n \rightarrow \infty} \int_{\Omega} \Delta u_{n} \Delta\left(u_{n} \phi_{\varepsilon}^{j}\right) d x .
$$

Then

$$
\left\{\begin{array}{l}
\lim _{\varepsilon \rightarrow 0} \int_{\Omega} \sum_{i=1}^{k} \phi_{\varepsilon}^{j} d \widetilde{\gamma}=\lim _{\varepsilon \rightarrow 0} \int_{\Omega} \sum_{i=1}^{k} \mu_{i} \frac{u_{0}^{2} \phi_{\varepsilon}^{j}}{\left|x-a_{i}\right|^{4}}=0, \\
\lim _{\varepsilon \rightarrow 0} \int_{\Omega} \phi_{\varepsilon}^{j} d \widetilde{v}=\lim _{\varepsilon \rightarrow 0}\left(\int_{\Omega}\left|u_{0}\right|^{2} \phi_{\varepsilon}^{j}+\widetilde{v_{x_{j}}}\right)=\widetilde{v}_{x_{j}}, \\
\lim _{\varepsilon \rightarrow 0} \lambda \int_{\Omega}\left|u_{0}\right|^{q} \phi_{\varepsilon}^{j} d x=0 .
\end{array}\right.
$$

On the other hand, by (4.2) and the weak convergence we can obtain

$$
\lim _{n \rightarrow \infty} \int_{\Omega} \Delta u_{n} \Delta\left(u_{n} \phi_{\varepsilon}^{j}\right) d x=\int_{\Omega} \phi_{\varepsilon}^{j} d \tilde{\mu}+\lim _{n \rightarrow \infty} \int_{\Omega} \Delta u_{n}\left(2 \nabla u_{n} \nabla \phi_{\varepsilon}^{j}+u_{n} \Delta \phi_{\varepsilon}^{j}\right) d x .
$$

Now, by (4.2) it is easy to see that

$$
\lim _{\varepsilon \rightarrow 0} \int_{\Omega} \phi_{\varepsilon}^{j} d \tilde{\mu} \geq \widetilde{\mu_{x_{j}}}
$$

By the Hölder inequality, we get

$$
\begin{aligned}
0 & \leq \varlimsup_{n \rightarrow \infty}\left|\int_{\Omega} \Delta u_{n}\left(\nabla u_{n} \nabla \phi_{\varepsilon}^{j}\right) d x\right| \\
& \leq \varlimsup_{n \rightarrow \infty}\left[\left(\int_{\Omega}\left|\Delta u_{n}\right|^{2} d x\right)^{\frac{1}{2}}\left(\int_{\Omega}\left|\nabla u_{n}\right|^{2}\left|\nabla \phi_{\varepsilon}^{j}\right|^{2} d x\right)^{\frac{1}{2}}\right] \\
& \leq C\left(\int_{B_{2 \varepsilon}\left(x_{j}\right)}\left|\nabla u_{0}\right|^{2}\left|\nabla \phi_{\varepsilon}^{j}\right|^{2} d x\right)^{\frac{1}{2}}
\end{aligned}
$$




$$
\begin{aligned}
& \leq C\left(\int_{B_{2 \varepsilon}\left(x_{j}\right)}\left|\nabla \phi_{\varepsilon}^{j}\right|^{N} d x\right)^{\frac{1}{N}}\left(\int_{B_{2 \varepsilon}\left(x_{j}\right)}\left|\nabla u_{0}\right|^{\frac{2 N}{N-2}} d x\right)^{\frac{N-2}{2 N}} \\
& \leq C\left(\int_{B_{2 \varepsilon}\left(x_{j}\right)}\left|\nabla u_{0}\right|^{\frac{2 N}{N-2}} d x\right)^{\frac{N-2}{2 N}} \rightarrow 0 \quad \text { as } \varepsilon \rightarrow 0
\end{aligned}
$$

and

$$
\begin{aligned}
0 & \leq \varlimsup_{n \rightarrow \infty}\left|\int_{\Omega} \Delta u_{n} u_{n} \Delta \phi_{\varepsilon}^{j} d x\right| \\
& \leq \varlimsup_{n \rightarrow \infty}\left[\left(\int_{\Omega}\left|\Delta u_{n}\right|^{2} d x\right)^{\frac{1}{2}}\left(\int_{\Omega}\left|\Delta \phi_{\varepsilon}^{j}\right|^{2}\left|u_{n}\right|^{2} d x\right)^{\frac{1}{2}}\right] \\
& \leq C\left(\int_{B_{2 \varepsilon}\left(x_{j}\right)}\left|\Delta \phi_{\varepsilon}^{j}\right|^{2}\left|u_{0}\right|^{2} d x\right)^{\frac{1}{2}} \\
& \leq C\left(\int_{B_{2 \varepsilon}\left(x_{j}\right)}\left|\Delta \phi_{\varepsilon}^{j}\right|^{\frac{N}{2}} d x\right)^{\frac{2}{N}}\left(\int_{B_{2 \varepsilon}\left(x_{j}\right)}\left|u_{0}\right|^{\frac{2 N}{N-4}} d x\right)^{\frac{N-4}{2 N}} \\
& \leq C\left(\int_{B_{2 \varepsilon}\left(x_{j}\right)}\left|u_{0}\right|^{2^{*}} d x\right)^{\frac{1}{2^{*}}} \rightarrow 0 \text { as } \varepsilon \rightarrow 0 .
\end{aligned}
$$

Thus, from (4.3)-(4.8) it follows that

$$
\widetilde{\mu_{x_{j}}} \leq \widetilde{v_{x_{j}}}
$$

By the Sobolev inequality, $S_{0} \widetilde{v_{x_{j}}} \frac{2}{2^{*}} \leq \widetilde{\mu_{x_{j}}}$, hence we deduce that

$$
\tilde{v_{x_{j}}}=0 \quad \text { or } \quad \tilde{v_{x_{j}}} \geq A_{0}^{\frac{N}{4}}
$$

which implies that $\mathcal{J}$ is finite. Claim 1 is proved.

Claim 2. We claim that

$$
\text { for each } i=1,2, \ldots, k \text { either } \quad \widetilde{v_{a_{i}}}=0 \quad \text { or } \quad \widetilde{v_{a_{i}}} \geq A_{\mu_{i}}^{\frac{N}{4}} .
$$

In order to prove claim 2 , for each $i=1,2, \ldots, k$, we consider the possibility of concentration at points $a_{i}(1 \leq i \leq k)$. For $\varepsilon>0$ be small enough such that $x_{j} \notin B_{\varepsilon}\left(a_{i}\right)$ for all $j \in \mathcal{J}$ and $B_{\varepsilon}\left(a_{i}\right) \cap B_{\varepsilon}\left(a_{j}\right)=\varnothing$ for $i \neq j$ and $1 \leq i, j \leq k$. Let $\varphi_{\varepsilon}^{i}$ be a smooth cut-off function centered at $a_{i}$ such that $0 \leq \varphi_{\varepsilon}^{i} \leq 1, \varphi_{\varepsilon}^{i}=1$ for $\left|x-a_{i}\right| \leq \varepsilon, \varphi_{\varepsilon}^{i}=0$ for $\left|x-a_{i}\right| \geq 2 \varepsilon,\left|\nabla \varphi_{\varepsilon}^{i}\right| \leq \frac{2}{\varepsilon}$ and $\left|\Delta \varphi_{\varepsilon}^{i}\right| \leq \frac{2}{\varepsilon^{2}}$. Then, by (4.2) and similar arguments to the proof of claim 1, we obtain

$$
\begin{aligned}
& \lim _{\varepsilon \rightarrow 0} \lim _{n \rightarrow \infty} \int_{\Omega} \Delta u_{n} \Delta\left(u_{n} \varphi_{\varepsilon}^{j}\right)=\lim _{\varepsilon \rightarrow 0} \int_{\Omega} \varphi_{\varepsilon}^{i} d \widetilde{\mu} \geq \lim _{\varepsilon \rightarrow 0}\left(\int_{\Omega}\left|\Delta u_{0}\right|^{2} \varphi_{\varepsilon}^{i}+\widetilde{\mu_{a_{i}}}\right)=\widetilde{\mu_{a_{i}}}, \\
& \lim _{\varepsilon \rightarrow 0} \lim _{n \rightarrow \infty} \int_{\Omega} \mu_{i} \frac{u_{n}^{2}}{\left|x-a_{i}\right|^{4}} \varphi_{\varepsilon}^{i}=\lim _{\varepsilon \rightarrow 0} \int_{\Omega} \varphi_{\varepsilon}^{i} d \widetilde{\gamma}=\lim _{\varepsilon \rightarrow 0}\left(\int_{\Omega} \mu_{i} \frac{u_{0}^{2}}{\left|x-a_{i}\right|^{4}} \varphi_{\varepsilon}^{i}+\widetilde{\gamma_{a_{i}}}\right)=\widetilde{r_{a_{i}}}, \\
& \lim _{\varepsilon \rightarrow 0} \lim _{n \rightarrow \infty} \int_{\Omega}\left|u_{n}\right|^{2^{*}} \varphi_{\varepsilon}^{i}=\lim _{\varepsilon \rightarrow 0} \int_{\Omega} \varphi_{\varepsilon}^{i} d \widetilde{v}=\lim _{\varepsilon \rightarrow 0}\left(\int_{\Omega}\left|u_{0}\right|^{2^{*}} \varphi_{\varepsilon}^{i}+\widetilde{v_{a_{i}}}\right)=\widetilde{v_{a_{i}}}, \\
& \lim _{\varepsilon \rightarrow 0} \lim _{n \rightarrow \infty} \int_{\Omega} \mu_{j} \frac{u_{n}^{2}}{\left|x-a_{j}\right|^{4}} \varphi_{\varepsilon}^{i}=0 \text { for } j \neq i .
\end{aligned}
$$


Thus we have

$$
0=\lim _{\varepsilon \rightarrow 0} \lim _{n \rightarrow \infty}\left\langle J_{\lambda}^{\prime}\left(u_{n}\right), u_{n} \varphi_{\varepsilon}^{i}\right\rangle \geq \widetilde{\mu_{a_{i}}}-\mu_{i} \widetilde{\gamma_{a_{i}}}-\widetilde{v_{a_{i}}} .
$$

From (4.5) and (4.6) we derive that $A_{\mu_{i}} \widetilde{v_{a_{i}}} \frac{2}{2^{*}} \leq \widetilde{v_{a_{i}}}$ for all $1 \leq i \leq k$, and then

$$
\text { either } \widetilde{v_{a_{i}}}=0 \text { or } \widetilde{v_{a_{i}}} \geq A_{\mu_{i}}^{\frac{N}{4}}
$$

Claim 2 is thereby proved.

From the above arguments and (4.1), we conclude that

$$
\begin{aligned}
c & =\lim _{n \rightarrow \infty}\left(J_{\lambda}\left(u_{n}\right)-\frac{1}{2}\left\langle J_{\lambda}^{\prime}\left(u_{n}\right), u_{n}\right\rangle\right) \\
& =\frac{2}{N} \lim _{n \rightarrow \infty} \int_{\Omega}\left|u_{n}\right|^{2^{*}}+\left(\frac{1}{2}-\frac{1}{q}\right) \lambda \int_{\Omega}\left|u_{0}\right|^{q} \\
& =\frac{2}{N}\left(\int_{\Omega}\left|u_{0}\right|^{2 *}+\sum_{j \in \mathcal{J}} \widetilde{v_{x_{j}}}+\sum_{i=1}^{k} \widetilde{v_{a_{i}}}\right)+\left(\frac{1}{2}-\frac{1}{q}\right) \lambda \int_{\Omega}\left|u_{0}\right|^{q} \\
& =\frac{2}{N}\left(\sum_{j \in \mathcal{J}} \widetilde{v_{x_{j}}}+\sum_{i=1}^{k} \widetilde{v_{a_{i}}}\right) .
\end{aligned}
$$

If $\widetilde{v_{a_{i}}}=\widetilde{v_{x_{j}}}=0$ for all $i \in\{1,2, \ldots, k\}$ and $j \in \mathcal{J}$, then $c=0$ which contradicts the assumption that $c>0$. On the other hand, if there exists an $i \in\{1,2, \ldots, k\}$ such that $\widetilde{v_{a_{i}}} \neq 0$ or there exists a $j \in \mathcal{J}$ with $\tilde{v_{j}} \neq 0$, then we infer that

$$
c \geq \frac{2}{N} \min \left\{A_{0}^{\frac{N}{4}}, A_{\mu_{1}}^{\frac{N}{4}}, A_{\mu_{2}}^{\frac{N}{4}}, \ldots, A_{\mu_{k}}^{\frac{N}{4}}\right\}=\frac{2}{N} A_{\mu_{k}}^{\frac{N}{4}},
$$

which also contradicts the assumption that $c<\frac{2}{N} A_{\mu_{k}}^{\frac{N}{4}}$. Therefore $u_{0}$ is a nonzero solution of Eq. $\left(E_{\lambda}\right)$.

Take $\delta_{0}>0$ small enough such that $B_{2 \delta_{0}}\left(a_{k}\right) \subset \Omega$. Choose the radial cut-off function $\eta(x)=\eta(|x|) \in C_{0}^{\infty}\left(B_{2 \delta_{0}}(0)\right)$ such that $0 \leq \eta(x) \leq 1$ in $B_{2 \delta_{0}}(0)$ and $\eta(x)=1$ in $B_{\delta_{0}}(0)$. Set $u_{\varepsilon}(x)=\eta\left(x-a_{k}\right) y_{\varepsilon}^{\mu_{k}}\left(x-a_{k}\right)$, where $y_{\varepsilon}^{\mu_{k}}(x)$ is the same function as in (1.2). The following asymptotic properties hold.

Lemma 4.3 Assume that $N \geq 5, \mu_{k} \in[0, \bar{\mu}), \delta=\frac{N-4}{2}$ and $1 \leq q<2 *$. Then, as $\varepsilon \rightarrow 0$, we have the following estimates:

$$
\begin{aligned}
& \int_{\Omega}\left(\left|\Delta u_{\varepsilon}\right|^{2}-\mu_{k} \frac{\left|u_{\varepsilon}\right|^{2}}{\left|x-a_{k}\right|^{4}}\right)=A_{\mu_{k}}^{\frac{N}{4}}+O\left(\varepsilon^{2\left(b\left(\mu_{k}\right)-\delta\right)}\right), \\
& \int_{\Omega}\left|u_{\varepsilon}\right|^{2^{*}}=A_{\mu_{k}}^{\frac{N}{4}}+O\left(\varepsilon^{2^{*}\left(b\left(\mu_{k}\right)-\delta\right)}\right),
\end{aligned}
$$


and

$$
\int_{\Omega}\left|u_{\varepsilon}\right|^{q}= \begin{cases}O_{1}\left(\varepsilon^{N-q \delta}\right), & \text { if } \frac{N}{b\left(\mu_{k}\right)}<q<2^{*} \\ O_{1}\left(\varepsilon^{N-q \delta}\right)|\ln \varepsilon|, & \text { if } q=\frac{N}{b\left(\mu_{k}\right)} \\ O_{1}\left(\varepsilon^{q\left(b\left(\mu_{k}\right)-\delta\right)}\right), & \text { if } 1 \leq q<\frac{N}{b\left(\mu_{k}\right)}\end{cases}
$$

Moreover, for all $N \geq 8$, as $\varepsilon \rightarrow 0$, we have

$$
\int_{\Omega}\left|u_{\varepsilon}\right|^{2}= \begin{cases}O_{1}\left(\varepsilon^{4}\right), & \text { if } 0 \leq \mu_{k}<\mu^{*} \\ O_{1}\left(\varepsilon^{4}|\ln \varepsilon|\right), & \text { if } \mu_{k}=\mu^{*}\end{cases}
$$

where $\mu^{*}:=\frac{1}{16}\left(N^{2}-16\right)\left(N^{2}-8 N\right)$.

Proof See Kang-Xu [19, Lemma 3.2].

Lemma 4.4 Let $N \geq 5,1 \leq q<2$ and assume that $(\mathcal{H})$ holds. Then, for any $\lambda>0$, there exists a $v_{\lambda} \in H_{0}^{2}(\Omega)$ such that

$$
\sup _{t \geq 0} J_{\lambda}\left(t v_{\lambda}\right)<\frac{2}{N} A_{\mu_{k}}^{\frac{N}{4}}
$$

In particular, $\alpha_{\lambda}^{-}<\frac{2}{N} A_{\mu_{k}}^{\frac{N}{4}}$ for all $\lambda \in\left(0, \Lambda_{0}\right)$.

Proof For $t \geq 0$, we consider the functions

$$
\begin{aligned}
g(t) & :=J_{\lambda}\left(t u_{\varepsilon}\right) \\
& =\frac{t^{2}}{2}\left\|u_{\varepsilon}\right\|^{2}-\frac{t^{2^{*}}}{2^{*}} \int_{\Omega}\left|u_{\varepsilon}\right|^{2^{*}}-\lambda \frac{t^{q}}{q} \int_{\Omega}\left|u_{\varepsilon}\right|^{q} \\
& \leq \frac{t^{2}}{2} \int_{\Omega}\left(\left|\Delta u_{\varepsilon}\right|^{2}-\mu_{k} \frac{u_{\varepsilon}^{2}}{\left|x-a_{k}\right|^{4}}\right)-\frac{t^{2^{*}}}{2^{*}} \int_{\Omega}\left|u_{\varepsilon}\right|^{2^{*}}-\lambda \frac{t^{q}}{q} \int_{\Omega}\left|u_{\varepsilon}\right|^{q}
\end{aligned}
$$

and

$$
\begin{aligned}
\bar{g}(t) & :=\frac{t^{2}}{2} \int_{\Omega}\left(\left|\Delta u_{\varepsilon}\right|^{2}-\mu_{k} \frac{u_{\varepsilon}^{2}}{\left|x-a_{k}\right|^{4}}\right)-\frac{t^{2^{*}}}{2^{*}} \int_{\Omega}\left|u_{\varepsilon}\right|^{2^{*}} \\
& =\frac{t^{2}}{2}\left\|u_{\varepsilon}\right\|_{\mu_{k}}^{2}-\frac{t^{2^{*}}}{2^{*}} \int_{\Omega}\left|u_{\varepsilon}\right|^{2^{*}},
\end{aligned}
$$

where $\left\|u_{\varepsilon}\right\|_{\mu_{k}}^{2}:=\int_{\Omega}\left(\left|\Delta u_{\varepsilon}\right|^{2}-\mu_{k} \frac{u_{\varepsilon}^{2}}{\left|x-a_{k}\right|^{4}}\right)$.

Using the definitions of $g$ and $u_{\varepsilon}$, we get

$$
g(t)=J_{\lambda}\left(t u_{\varepsilon}\right) \leq \frac{t^{2}}{2}\left\|u_{\varepsilon}\right\|_{\mu_{k}}^{2}, \quad \text { for all } t \geq 0 \text { and } \lambda>0
$$

Combining this with (4.9), let $\varepsilon \in(0,1)$, then there exists $t_{0} \in(0,1)$ not depending on $\varepsilon$ such that

$$
\sup _{0 \leq t \leq t_{0}} g(t)<\frac{2}{N} A_{\mu_{k}}^{\frac{N}{4}}, \quad \text { for all } \lambda>0 \text { and } \varepsilon \in(0,1) .
$$


On the other hand, by the fact that

$$
\max _{t \geq 0}\left(\frac{t^{2}}{2} B_{1}-\frac{t^{2^{*}}}{2^{*}} B_{2}\right)=\frac{2}{N} B_{1}^{\frac{N}{4}} B_{2}^{\frac{4-N}{4}}, \quad B_{1}>0, B_{2}>0,
$$

and by (4.9) and (4.10), we can get

$$
\begin{aligned}
\max _{t \geq 0} \bar{g}(t) & =\frac{2}{N}\left\|u_{\varepsilon}\right\|_{\mu_{k}}^{\frac{N}{4}}\left(\int_{\Omega}\left|u_{\varepsilon}\right|^{2^{*}}\right)^{\frac{4-N}{4}} \\
& =\frac{2}{N}\left(A_{\mu_{k}}^{\frac{N}{4}}+O\left(\varepsilon^{2\left(b\left(\mu_{k}\right)-\delta\right)}\right)\right)^{\frac{N}{4}}\left(A_{\mu_{k}}^{\frac{N}{4}}+O\left(\varepsilon^{2^{*}\left(b\left(\mu_{k}\right)-\delta\right)}\right)\right)^{\frac{4-N}{4}} \\
& =\frac{2}{N} A_{\mu_{k}}^{\frac{N}{4}}+O\left(\varepsilon^{2\left(b\left(\mu_{k}\right)-\delta\right)}\right) .
\end{aligned}
$$

Hence, for all $\lambda>0,1 \leq q<2$, by (4.15) we have

$$
\begin{aligned}
\sup _{t \geq t_{0}} g(t) & \leq \sup _{t \geq t_{0}}\left(\bar{g}(t)-\lambda \frac{t^{q}}{q} \int_{\Omega}\left|u_{\varepsilon}\right|^{q}\right) \\
& \leq \frac{2}{N} A_{\mu_{k}}^{\frac{N}{4}}+O\left(\varepsilon^{2\left(b\left(\mu_{k}\right)-\delta\right)}\right)-\lambda \frac{t_{0}^{q}}{q} \int_{\Omega}\left|u_{\varepsilon}\right|^{q} .
\end{aligned}
$$

Now, we need to distinguish two cases.

Case (i): $1 \leq q<\frac{N}{b(\mu)}$ and $q<2$. By (1.3) and (4.11) we have as $\varepsilon \rightarrow 0$

$$
\int_{\Omega}\left|u_{\varepsilon}\right|^{q}=O_{1}\left(\varepsilon^{q(b(\mu)-\delta)}\right)>O\left(\varepsilon^{2(b(\mu)-\delta)}\right) .
$$

Combining this with (4.14) and (4.16), for any $\lambda>0$, we can choose $\varepsilon_{\lambda}$ small enough such that

$$
\sup _{t \geq 0} J_{\lambda}\left(t u_{\varepsilon_{\lambda}}\right)<\frac{2}{N} A_{\mu_{k}}^{\frac{N}{4}}
$$

Case (ii): $\frac{N}{b(\mu)} \leq q<2$. By (4.11) we have

$$
\int_{\Omega}\left|u_{\varepsilon}\right|^{q}= \begin{cases}O_{1}\left(\varepsilon^{N-q \delta}\right), & \text { if } q>\frac{N}{b(\mu)} \\ O_{1}\left(\varepsilon^{N-q \delta}|\ln \varepsilon|\right), & \text { if } q=\frac{N}{b(\mu)}\end{cases}
$$

Moreover, it follows from $b(\mu)>\delta$ and $q \geq \frac{N}{b(\mu)}$ that

$$
2(b(\mu)-\delta)>q(b(\mu)-\delta) \geq N-q \delta .
$$

Combining this with (4.14) and (4.16), for any $\lambda>0$, we can choose $\varepsilon_{\lambda}$ small enough such that

$$
\sup _{t \geq 0} J_{\lambda}\left(t u_{\varepsilon_{\lambda}}\right)<\frac{2}{N} A_{\mu_{k}}^{\frac{N}{4}}
$$


From cases (i) and (ii), (4.13) holds by taking $v_{\lambda}=u_{\varepsilon_{\lambda}}$.

From Lemma 2.4, the definition of $\alpha_{\lambda}^{-}$and (4.13), for any $\lambda \in\left(0, \Lambda_{0}\right)$, we see that there exists $t_{\lambda}^{-}>0$ such that $t_{\lambda}^{-} v_{\lambda} \in \mathcal{M}_{\lambda}^{-}$and

$$
\alpha_{\lambda}^{-} \leq J_{\lambda}\left(t_{\lambda}^{-} v_{\lambda}\right) \leq \sup _{t \geq 0} J_{\lambda}\left(t v_{\lambda}\right)<\frac{2}{N} A_{\mu_{k}}^{\frac{N}{4}} .
$$

The proof is thus completed.

Now, we establish the existence of a local minimum of $J_{\lambda}$ on $\mathcal{M}_{\lambda}^{-}$.

Theorem 4.5 Assume that $N \geq 5$ and the condition $(\mathcal{H})$ holds. If $\lambda \in\left(0, \frac{q}{2} \Lambda_{0}\right)$, then $J_{\lambda}$ has a minimizer $U_{\lambda}$ in $\mathcal{M}_{\lambda}^{-}$and such that:

(i) $J_{\lambda}\left(U_{\lambda}\right)=\alpha_{\lambda}^{-}$.

(ii) $U_{\lambda}$ is a nontrivial solution of $E q$. $\left(E_{\lambda}\right)$.

Proof If $\lambda \in\left(0, \frac{q}{2} \Lambda_{0}\right)$, then, by Lemma 3.1(ii), Proposition 3.4(ii) and Lemma 4.4, there ex-

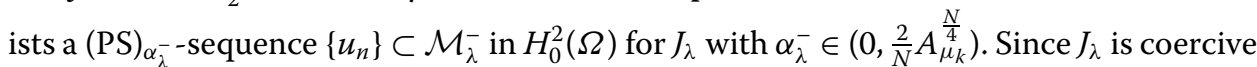
on $\mathcal{M}_{\lambda}$ (see Lemma 4.1), we see that $\left\{u_{n}\right\}$ is bounded in $H_{0}^{2}(\Omega)$. From Lemma 4.2, there exist a subsequence still denoted by $\left\{u_{n}\right\}$ and a nonzero solution $U_{\lambda} \in H_{0}^{2}(\Omega)$ of Eq. $\left(E_{\lambda}\right)$ such that $u_{n} \rightarrow U_{\lambda}$ weakly in $H_{0}^{2}(\Omega)$.

Now, we first prove that $U_{\lambda} \in \mathcal{M}_{\lambda}^{-}$. Arguing by contradiction, we assume $U_{\lambda} \in \mathcal{M}_{\lambda}^{+}$. Then, by Lemma 2.4 , there exists a unique $t_{\lambda}^{-}$such that $t_{\lambda}^{-} U_{\lambda} \in \mathcal{M}_{\lambda}^{-}$. It follows that

$$
\alpha_{\lambda}^{-} \leq J_{\lambda}\left(t_{\lambda}^{-} U_{\lambda}\right)<\lim _{n \rightarrow \infty} J_{\lambda}\left(t_{\lambda}^{-} u_{n}\right) \leq \lim _{n \rightarrow \infty} J_{\lambda}\left(u_{n}\right)=\alpha_{\lambda}^{-} .
$$

This is a contradiction. Consequently, $U_{\lambda} \in \mathcal{M}_{\lambda}^{-}$.

Next, by the same argument as that in Theorem 3.5 , we get $u_{n} \rightarrow U_{\lambda}$ strongly in $H_{0}^{2}(\Omega)$ and $J_{\lambda}\left(U_{\lambda}\right)=\alpha_{\lambda}^{-}>0$ for all $\lambda \in\left(0, \frac{q}{2} \Lambda_{0}\right)$. Since $J_{\lambda}\left(U_{\lambda}\right)=J_{\lambda}\left(\left|U_{\lambda}\right|\right)$ and $\left|U_{\lambda}\right| \in \mathcal{M}_{\lambda}^{-}$, by Lemma 2.2 we may assume that $U_{\lambda}$ is a nontrivial nonnegative solution of Eq. $\left(E_{\lambda}\right)$. The proof of this theorem is then completed.

Proof of Theorem 1.1 The part (i) of Theorem 1.1 immediately follows from Theorem 3.5. When $0<\lambda<\frac{q}{2} \Lambda_{0}<\Lambda_{0}$, by Theorems 3.5, and 4.5, we see that Eq. $\left(E_{\lambda}\right)$ has at least two nontrivial solutions $u_{\lambda}$ and $U_{\lambda}$ such that $u_{\lambda} \in \mathcal{M}_{\lambda}^{+}$and $U_{\lambda} \in \mathcal{M}_{\lambda}^{-}$. Since $\mathcal{M}_{\lambda}^{+} \cap \mathcal{M}_{\lambda}^{-}=\emptyset$, this implies that $u_{\lambda}$ and $U_{\lambda}$ are distinct. This completes the proof of Theorem 1.1.

\section{Existence of solutions in the case of $2 \leq q<2^{*}$}

In order to prove Theorem 1.2, we first establish several lemmas.

Lemma 5.1 Let $N \geq 5$ and assume that $(\mathcal{H})$ holds and one of the following conditions hold:

(i) $\lambda>0,2<q<2^{*}$.

(ii) $0<\lambda<\lambda_{1}, q=2$.

Then the functional $J_{\lambda}$ satisfies the (PS) condition for all $c<c^{*}:=\frac{2}{N} A_{\mu_{k}}^{\frac{N}{4}}$.

Proof The argument is standard and is omitted (e.g. [17]) 
Lemma 5.2 Let $N \geq 5$ and assume that $(\mathcal{H})$ holds and one of the following conditions holds:

(i) $\lambda>0, \bar{q}<q<2^{*}$, where

$$
\bar{q}=\max \left\{2, \frac{N}{b\left(\mu_{k}\right)}, \frac{4\left(N-2-b\left(\mu_{k}\right)\right)}{N-4}\right\} .
$$

(ii) $N \geq 8,0<\lambda<\lambda_{1}, q=2,0 \leq \mu_{k} \leq \mu^{*}$.

Then as $\varepsilon \rightarrow 0^{+}$we have

$$
\sup _{t \geq 0} J_{\lambda}\left(t u_{\varepsilon}\right)<c^{*}=\frac{2}{N} A_{\mu_{k}}^{\frac{N}{4}}
$$

where $\lambda_{1}$ is the same as in (1.6) and $u_{\varepsilon}$ is the same function as in Lemma 4.3.

Proof For $t \geq 0$, we define the functions $g(t):=J_{\lambda}\left(t u_{\varepsilon}\right)$ and

$$
\bar{g}(t):=\frac{t^{2}}{2} \int_{\Omega}\left(\left|\Delta u_{\varepsilon}\right|^{2}-\mu_{k} \frac{u_{\varepsilon}^{2}}{\left|x-a_{k}\right|^{4}}\right)-\frac{t^{2^{*}}}{2^{*}} \int_{\Omega}\left|u_{\varepsilon}\right|^{2^{*}} .
$$

(i) Since $\lambda>0,2<q<2^{*}$, a direct calculation shows that $\sup _{t \geq 0} g(t)$ can be obtained at finite $t_{\varepsilon}>0$ such that

$$
0=g^{\prime}\left(t_{\varepsilon}\right)=t_{\varepsilon}\left(\left\|u_{\varepsilon}\right\|^{2}-t_{\varepsilon}^{2^{*}-2} \int_{\Omega}\left|u_{\varepsilon}\right|^{2^{*}}-\lambda t_{\varepsilon}^{q-2} \int_{\Omega}\left|u_{\varepsilon}\right|^{q}\right)
$$

Furthermore, $t_{\varepsilon} \in\left[C_{1}, C_{2}\right]$, where $C_{1}$ and $C_{2}$ are positive constants independent of $\varepsilon$.

From the definitions of $g, \bar{g}$ and (4.15), it follows that

$$
g\left(t_{\varepsilon}\right) \leq \bar{g}\left(t_{\varepsilon}\right)-\frac{\lambda}{q} t_{\varepsilon}^{q} \int_{\Omega}\left|u_{\varepsilon}\right|^{q} \leq c^{*}+O\left(\varepsilon^{2\left(b\left(\mu_{k}\right)-\delta\right)}\right)-C \int_{\Omega}\left|u_{\varepsilon}\right|^{q} .
$$

If $\bar{q}<q<2^{*}$, by (4.11) we have

$$
\int_{\Omega}\left|u_{\varepsilon}\right|^{q}=O_{1}\left(\varepsilon^{N-q \delta}\right)
$$

Since $\left.2\left(b\left(\mu_{k}\right)-\delta\right)\right)>N-q \delta$, from (5.2) and (5.3) it follows that

$$
\sup _{t \geq 0} J_{\lambda}\left(t u_{\varepsilon}\right)=g\left(t_{\varepsilon}\right)<c^{*}
$$

(ii) Suppose that $N \geq 8,0<\lambda<\lambda_{1}, q=2,0 \leq \mu_{k} \leq \mu^{*}$. A direct calculation shows that

$$
0 \leq \mu_{k} \leq \mu^{*} \quad \Longleftrightarrow b\left(\mu_{k}\right)-\delta>2, \quad \mu_{k}=\mu^{*} \quad \Longleftrightarrow \quad b\left(\mu_{k}\right)-\delta=2 .
$$

Using a similar argument to (i), we can deduce that $\sup _{t \geq 0} g(t)<c^{*}$ is attained at finite $t_{\varepsilon}>0$. Moreover,

$$
g\left(t_{\varepsilon}\right) \leq \bar{g}\left(t_{\varepsilon}\right)-\lambda \frac{t_{\varepsilon}^{2}}{2} \int_{\Omega}\left|u_{\varepsilon}\right|^{2} \leq c^{*}+O\left(\varepsilon^{2\left(b\left(\mu_{k}\right)-\delta\right)}\right)-C \int_{\Omega}\left|u_{\varepsilon}\right|^{2} .
$$


Then by (4.12) and (5.4) it follows that (5.1) holds as $\varepsilon \rightarrow 0^{+}$. The proof is thus completed.

Proof of Theorem 1.2 According to Lemmas 5.1 and 5.2 and applying the mountain-pass theorem $[2,4]$, Theorem 1.2 can be concluded to.

\section{Acknowledgements}

The authors would like to thank the referees for their valuable comments and suggestions which improved the original manuscript.

Funding

T.S. Hsu was supported by the Ministry of Science and Technology, Taiwan (Grant No. 107-2115-M-182-002-).

Availability of data and materials

Not applicable.

Competing interests

The authors declare that they have no competing interests.

\section{Authors' contributions}

All authors have contributed equally to the paper. All authors read and approved the final version of the manuscript.

\section{Author details}

${ }^{1}$ School of Mathematics, Jiangxi Normal University, Nanchang, P.R. China. ${ }^{2}$ Department of Natural Science in the Center for General Education, Chang Gung University, Taoyuan, Taiwan.

\section{Publisher's Note}

Springer Nature remains neutral with regard to jurisdictional claims in published maps and institutional affiliations.

Received: 9 January 2019 Accepted: 22 May 2019 Published online: 30 May 2019

\section{References}

1. Alves, C.O., do Ó, J.M.: Positive solutions of a fourth-order semilinear problem involving critical growth. Adv. Nonlinear Stud. 2(4), 437-458 (2002)

2. Ambrosetti, A., Rabinowitz, H.: Dual variational methods in critical point theory and applications. J. Funct. Anal. 14 349-381 (1973)

3. Bhakta, M., Musina, R.: Entire solutions for a class of variational problems involving the biharmonic operator and Rellich potentials. Nonlinear Anal. 75(9), 3836-3848 (2012)

4. Brézis, H., Nirenberg, L.: Positive solutions of nonlinear elliptic equations involving critical Sobolev exponents. Commun. Pure Appl. Math. 36, 437-477 (1983)

5. Brown, K.J., Zhang, Y.: The Nehari manifold for a semilinear elliptic equation with a sign-changing weigh function. J. Differ. Equ. 193, 481-499 (2003)

6. Caldiroli, P.: Radial and non radial ground states for a class of dilation invariant fourth order semilinear elliptic equations on $\mathbb{R}^{N}$. Commun. Pure Appl. Anal. 13(2), 811-821 (2014)

7. Caldiroli, P., Musina, R.: Caffarelli-Kohn-Nirenberg type inequalities for the weighted biharmonic operator in cones. Milan J. Math. 79, 657-687 (2011)

8. Catrina, F., Wang, Z.-Q.: On the Caffarelli-Kohn-Nirenberg inequalities: sharp constants, existence (and nonexistence), and symmetry of extremal functions. Commun. Pure Appl. Math. 54, 229-258 (2001)

9. Coffman, C.V.: On the structure of solutions to $\Delta^{2} u=\lambda u$ which satisfy the clamped plate conditions on a right angle. SIAM J. Math. Anal. 13, 746-757 (1982)

10. D'Ambrosio, L., Jannelli, E.: Nonlinear critical problems for the biharmonic operator with Hardy potential. Calc. Var. Partial Differ. Equ. 54, 365-396 (2015)

11. Edmunds, D., Fortunato, D., Jannelli, E.: Critical exponents, critical dimensions and the biharmonic operator. Arch. Ration. Mech. Anal. 112, 269-289 (1990)

12. Ekeland, I.: On the variational principle. J. Math. Anal. Appl. 47, 324-353 (1974)

13. Gazzola, F., Grunau, H.C.: Radial entire solutions for supercritical biharmonic equations. Math. Ann. 334, 905-936 (2006)

14. Hsu, T.S.: Multiplicity results for $p$-Laplacian with critical nonlinearity of concave-convex type and sign-changing weight functions. Abstr. Appl. Anal. 2009, 1 (2009)

15. Hsu, T.S.: Multiple positive solutions for semilinear elliptic equations involving multi-singular inverse square potentials and concave-convex nonlinearities. Nonlinear Anal. 74, 3703-3715 (2011)

16. Hsu, T.S., Zhang, J.: Multiple nontrivial solutions for critical biharmonic problems involving Rellich-type potentials and concave-convex nonlinearities. Preprint

17. Kang, D.: On the quasilinear elliptic problems with critical Sobolev-Hardy exponents and Hardy terms. Nonlinear Anal. 68, 1973-1985 (2008)

18. Kang, D., Xiong, P.: Ground state solutions to biharmonic equations involving critical nonlinearities and multiple singular potentials. Appl. Math. Lett. 66, 9-15 (2017) 
19. Kang, D., Xu, L.: Asymptotic behavior and existence results for the biharmonic problems involving Rellich potentials. J. Math. Anal. Appl. 455, 1365-1382 (2017)

20. Lions, P.L.: The concentration compactness principle in the calculus of variations, the limit case (I). Rev. Mat. Iberoam. $1(1), 145-201$ (1985)

21. Lions, P.L.: The concentration compactness principle in the calculus of variations, the limit case (II). Rev. Mat. Iberoam. $1(2), 45-121(1985)$

22. Rellich, F: Perturbation Theory of Eigenvalue Problems. Courant Institute of Mathematical Sciences, New York University, New York (1954)

Submit your manuscript to a SpringerOpen ${ }^{\circ}$ journal and benefit from:

- Convenient online submission

- Rigorous peer review

- Open access: articles freely available online

- High visibility within the field

- Retaining the copyright to your article

Submit your next manuscript at $\gg$ springeropen.com 